\title{
La medida de la empleabilidad en las empresas de inserción en el País Vasco: de la exclusión a la inserción sociolaboral
}

\author{
Lucía I. Llinares Insa
}

Departamento de Psicología Social, Facultad de Psicología, Universitat de València <lucia.llinares@uv.es>

\author{
Ana I. Córdoba Iñesta \\ Departamento de Psicología Evolutiva y de la Educación, Facultad de Psicología, \\ Universitat de València
}

\section{Juan José Zacarés González}

Departamento de Psicología Evolutiva y de la Educación, Facultad de Psicología, Universitat de València

\begin{abstract}
Azterlan honetan aurkezten da Euskadiko gizarteratzeko enpresen ibilbideetan agertzen diren enplegagarritasunerako politikaren inguruko ikusmoldeak. Gaiak garrantzia du laneko bazterketa bidezko egiten laguntzen duelako. Azken batean, banakoaren arduraren arloko gaia baita ahulezia bizi duten kolektiboen egoera hori; izan ere, gaitasun eta trebezia batzuen gabeziek sortzen baitute langabezia haztaraztea. Errealitatearen interpretazio hori baliabide gertatzen da gizarte-ekintzaren gidalerro eta ebaluazioen oinarri gisara. Lan hori burutzeko aztertu dira gizarteratzeko enpresetan enplegagarritasunaren elementuak ebaluatzeko erabiltzen diren tresnak. Artikulu honen bidez, gogoeta piztu nahi da errealitatearen interpretazio horren eta egunerokoan duen berroren eraginaz; eta, horrela, partaide izan nahi da laneratzea praktikan gidatzen duen maila sinboliko hori zuzentzeko gizarteratzeko enpresa horiek elkarlanean egindako ahaleginean.
\end{abstract}

\section{HITZ-GAKOAK:}

laneratze eta gizarteratzea, sartzeko ibilbideak, enplegagarritasuna, gizarte-bazterketa, gizarteratzeko enpresa.
Este artículo presenta un análisis de las concepciones de la empleabilidad que están presentes en los itinerarios de las empresas de inserción del País Vasco. Su importancia reside en ser el constructo que justifica la exclusión laboral: la situación en la que se encuentran los colectivos vulnerables es responsabilidad individual, ya que el desempleo es causa de la falta de determinadas competencias y habilidades. Esta interpretación de la realidad sirve de eje de evaluación y guía para la acción social. Para elaborar este trabajo, se han analizado los elementos de empleabilidad que se evalúan en los instrumentos utilizados en las empresas de inserción. El artículo trata de suscitar una reflexión sobre los modos de interpretar la realidad y su influencia en la práctica diaria, y participar así en la acción conjunta, emprendida por las empresas de inserción, dirigida a corregir el mundo simbólico que rige la práctica de la inserción laboral.

\section{Palabras ClaVe:}

inserción sociolaboral, itinerarios de inserción, empleabilidad, exclusión social, empresa de inserción. 


\section{Introducción}

Estamos de acuerdo con Álvarez-Uría cuando dice que, desde finales de los años setenta, la retórica neoliberal se ha impuesto de forma acrítica "como si se tratara de una verdad revelada" (2002: 15). Ésta tiene un nombre clave, la empleabilidad, y una repercusión inmediata, 'el aumento de la exclusión'. Ahora bien, el discurso de la empleabilidad no se introduce por implosión; se desarrolla en un marco laboral cambiante que pasa del Estado del bienestar a la era neoliberal, a través de etapas sucesivas, pero intrusivas, en las lógicas sociales y en las organización de la vida de las personas. Estos cambios paulatinos y continuos se constatan en las políticas económicas y en la organización de las empresas, y derivan en cambios en el mercado de trabajo y en las bases que los sustentan. Dichos cambios, por supuesto, tienen como elemento discursivo la empleabilidad.

La empleabilidad se puede analizar desde diferentes perspectivas (Llinares, Córdoba y Zacarés, 2011). Desde una perspectiva posibilista, se refiere a la capacidad que tienen algunas personas para obtener y mantener el empleo, cambiar de empleo o progresar en la misma organización (Campos Ríos, 2003; Finn, 2000; Formichella y London, 2005; McQuaid y Lyndsay, 2005). Esta visión convierte a la persona en responsable de su situación sociolaboral e implica dotar a la persona de las competencias dinámicas consideradas relevantes en el mundo laboral, y de estrategias de búsqueda de empleo. Por tanto, desde esta perspectiva, las desigualdades se interpretan como incapacidad para aprovechar las oportunidades laborales, y no como desigualdad real de posición social (Santos Ortega, 2003). El marco que lo acoge se fundamenta en la reducción de los niveles de protección y reglamentación laboral legal, y en el uso libre y económico de los recursos humanos, todo ello sobre la base de la necesidad del ajuste flexible y competitivo de las empresas (Brunet y Pastor, 2003). El fin de las políticas públicas se dirige, pues, a incrementar la seguridad del beneficio mercantil, la competitividad del tejido productivo, la adaptación del capital humano y la formación tecnológica (Mingione, 1994; Prieto, 1999b). Las políticas de ocupación, por su parte, se basan en el uso libre y lo más económico posible de los recursos humanos (Alaluf y Martínez, 1999; Prieto, 1999a). En ellas, la producción de bienes económicos pasa a ser el objetivo prioritario (Noguera, 2002).

La empleabilidad también genera un nuevo marco en las relaciones laborales empresario-trabajador. De este modo, al recaer la responsabilidad de su situación profesional en el trabajador, la carrera laboral, que anteriormente se concebía como la formación en un único oficio y en una única empresa, desaparece y pasa a considerarse clave la libertad del trabajador en la construcción de su futuro (Clarke y Patrickson, 2008). Es la persona la que acumula capital humano a partir de una decisión individual de optimización y de un balance entre los costes y los beneficios; y es ella quien tiene la responsabilidad de generar su currículo (Kippenberger, 2000) y de mejorar su empleabilidad (Fugate, Kinicki y Ashforth, 2004). Así, la autogestión de su carrera proporciona al trabajador un reparto justo para el futuro y mayor probabilidad de éxito (Baruch y Peiperl, 2000).

Ahora bien, desde una perspectiva alternativa, la perspectiva crítica, esto es una falacia en un contexto en el que el mercado laboral aparece segmentado (Casal, 2003). Actualmente, nos encontramos con un mercado primario que ofrece empleos estables y condiciones aceptables de trabajo y promoción, porque se mantienen las antiguas relaciones laborales en vías de extinción, aunque los recursos humanos tienen exceso de trabajo. Junto a él, existe un mercado secundario, que se caracteriza por ofrecer puestos de trabajo precarios e inestables, y malas condiciones de trabajo (De la Fuente y González-Castro, 2009). Tal y como indica Pérez (2003), la diferencia de pertenencia a uno u otro segmento no depende de la inversión en capital humano, sino de otros factores sociales que determinan la inclusión. La tendencia es la desaparición del primero en vías a la consecución del segundo, y la primacía de la movilidad laboral entre empresas y, por tanto, el continuo cambio de empleo (De Cuyper et al., 2011; Forrier, Sels y Stynen, 2009; Rothery y Roberstson, 1995) gracias a las nuevas modalidades de ocupación temporal y de facilidad de despido (Alaluf y Martínez, 1999; Prieto, 1999). Esto supone un cambio en las condiciones de contratación en varios sentidos (Añez, 2004; Capelli, 1995; Harrison, 1998; Macía, 2003; Osterman, 1994): por una parte, se reduce el salario del capital productivo y, por otra, las contrataciones se regulan por las necesidades de la empresa. Junto a ello, nos encontramos con otra contradicción: para Tezanos (2001), el proceso de degradación del trabajo no sólo supone el intercambio de trabajadores en las empresas y el empobrecimiento de la población, sino también el aumento del desempleo estructural de larga duración.

La perspectiva crítica también pone de manifiesto que, desde la perspectiva posibilista, que es la que legitima el sistema laboral actual, el problema del desempleo se percibe como la falta de ajuste entre la oferta de trabajo y las características de los demandantes de empleo. Así pues, desde esta última perspectiva la empleabilidad legitima lo que hace el mercado, y lo hace sobre la base de la responsabilidad personal de la situación social que viven las personas. Es, pues, el mérito de cada individuo, y no un proceso social, el que configura la representación social de las diferencias laborales de las personas, lo que justifica que los salarios se determinen sobre la base de las aportaciones del trabajador y, por tanto, sobre sus capacidades (Thurow, 2000). Esta diferenciación es percibida por la perspectiva crítica como debilitadora y tiende a excluir a parte de la población del sistema normalizado (Luttwak, 200o). Acrecienta, además, las dificultades de inserción laboral en el mercado reglado de los grupos poblacionales desfavorecidos y en riesgo de exclusión social, ya que los más desfavorecidos tienen menos oportunidades 
para adquirir competencias de empleabilidad, lo que determina que continúen en las capas más pobres y no sean empleables (Weinberg, 2004).

Castells (2001) conceptualiza la exclusión social como un proceso por el cual a ciertos individuos y grupos se les impide acceder a unas posiciones sociales. De esta forma, ciertas personas o grupos son excluidos de determinadas redes sociales, económicas o culturales (De la Fuente y GonzálezCastro, 2009). En este contexto, se diferencia entre ser empleable y no empleable, y esta bipolaridad etiqueta a las personas en función de su capacidad para conseguir un empleo (Finn, 2000; Van der Heijden y Van der Heijden, 2005).

Si el fin último es conseguir y mantener el empleo, y éste se debe a las competencias y habilidades de la persona, desde el discurso posibilista y legitimador del sistema laboral actual, la inversión que se realice para aumentar la empleabilidad hará disminuir las tasas de desempleo (Weinberg, 2004). Ello conlleva la generación de acciones e intervenciones individualizadas destinadas a aumentar la empleabilidad de los grupos desfavorecidos, a través de una combinación de actividades de formación y búsqueda de empleo (McQuaid y Lindsay, 2005). Es por ello por lo que, actualmente, la empleabilidad no sólo ocupa un lugar central en las políticas de mercado, sino que es uno de los cuatro pilares de la Estrategia Europea de Empleo (Comisión Europea, 1999). La Comisión Europea tiene el objetivo de promocionar el capital humano por medio de medidas activas y preventivas para los desempleados (Comisión Europea, 2003). Se parte de la creencia de que la empleabilidad que tiene una persona se debe a la inversión social que le permite acceder a ellas y, por tanto, es responsabilidad del sujeto defender ese patrimonio (Weinberg, 2004). La finalidad de la intervención, por tanto, es la integración/inserción sociolaboral con el fin de garantizar una participación activa en la vida social.

La inserción laboral es un territorio muy amplio y difuso: contempla todos los procesos, las herramientas, los análisis del mercado laboral y las metodologías de acceso al empleo que se dirigen a las personas que desean participar en el mundo laboral (De la Fuente y González-Castro, 2009). Los servicios sociales y algunas asociaciones sin ánimo de lucro ofrecen determinados colectivos a profesionales (psicólogos, pedagogos, educadores sociales y otros expertos) que analizan la empleabilidad de los desempleados y proponen programas de intervención, combinando medidas básicas de socialización, cursos de formación, periodos de prácticas o experiencias ocupacionales (Santos Ortega, 2003). Una de las iniciativas actuales más demandadas para el periodo formativo-laboral profesional previo, y que se percibe como clave en la sociedad actual, por su aportación a la inserción sociolaboral, es la empresa de inserción.

Las empresas de inserción llevan a cabo un acompañamiento individualizado del sujeto en la inserción laboral de las personas (González Barriga, 2003).
Son empresas que realizan una actividad económica planificada en el mercado de bienes y servicios, con la finalidad de proporcionar puestos de trabajo a personas en situación o riesgo de exclusión social (González Barriga, 2003); su objetivo es contratar a personas con un nivel de empleabilidad básico y suficiente para desempeñar un puesto de trabajo, pero no suficiente para su inclusión en el mercado de trabajo normalizado (Marhuenda, Bernad y Navas, 2010). Son, pues, un contexto propicio para trabajar la formación en un oficio concreto, y la interiorización y el desarrollo de los indicadores de empleabilidad requeridos en el mundo laboral normalizado, considerados como elementos indispensables de la normalidad laboral actual (Marhuenda, 2009; Santos Ortega, 2003).

\section{El estudio sobre los indicadores de empleabilidad}

Al igual que cualquier otra entidad que pretende la inserción sociolaboral, las empresas de inserción evalúan la empleabilidad de las personas y fijan itinerarios de formación personalizados. En ellas, la empleabilidad se mide en tres momentos: acogida, seguimiento, y transición o tránsito al mercado laboral ordinario.

- La fase de acogida, también denominada de incorporación o de evaluación inicial, es una fase de comunicación y conocimiento de expectativas mutuas entre el trabajador y la empresa. En ella se lleva a cabo la valoración inicial, dentro de la cual se enmarca el diagnóstico de empleabilidad. A partir de diversos procedimientos de evaluación (entrevistas, dinámicas grupales, aplicación de cuestionarios y pruebas estandarizadas, y observación, entre otras), se elabora un itinerario de inserción personalizado. De la evaluación anterior surge una propuesta de intervención, que trata de incidir en las causas, más que en los síntomas, de las limitaciones o carencias detectadas. Esta propuesta se concreta en el diseño, junto con el trabajador, de su propio itinerario de inserción.

- La fase de seguimiento, o de permanencia y acompañamiento en la empresa de inserción, es la de mayor duración y tiene como objetivo acompañar a la persona en un puesto de trabajo real. En ella se lleva a cabo una evaluación continua de la actuación en el trabajo, y de revisión y mejora del plan de trabajo trazado.

- Por último, el tercer momento es el de transición al mercado laboral ordinario, o de finalización y apoyo a la inserción sociolaboral. En él se evalúa si se han alcanzado gran parte de los objetivos acordados en el plan de trabajo y se determina si el proceso de inserción ha finalizado. Ésta es una de las fases más complejas si se producen resistencias por parte del trabajador a abandonar la empresa. Tales resistencias pueden interpretarse tanto como temores del propio trabajador hacia un entorno ‘menos protegido’ como, paradójica- 
mente, un indicador de buen funcionamiento de la empresa de inserción, al ajustarse a las necesidades personales del trabajador. Resulta adecuado asumir dichas resistencias como una nueva posibilidad para el trabajo educativo, pues exige revisar las expectativas y la empleabilidad inicial, y compararlas con la representación que la persona en inserción y que los agentes sociales de la empresa se hacen de su situación previa a la salida de la empresa de inserción. Las concepciones teóricas y las representaciones sobre la empleabilidad y los elementos que la constituyen que tienen estos agentes sociales son el objetivo del trabajo que aquí se presenta (Zacarés et al., 2011).

Para analizar las representaciones sobre la empleabilidad de los agentes sociales implicados en las empresas de inserción, se han identificado y clasificado los elementos de empleabilidad que se evalúan en sus instrumentos, a modo de indicadores. Como preparación previa del I Encuentro Estatal de Trabajadores/as Acompañantes, Técnicos/as de Acompañamiento y Trabajadores/as de Producción, organizado por la Federación de Asociaciones Empresariales de Empresas de Inserción (FAEDEI) y la Asociación Española de Recuperadores de Economía Social y Solidaria (AERESS), se solicitó a las empresas de inserción participantes diversos documentos sobre su práctica profesional. Entre dichos documentos, se hallaban los instrumentos y protocolos que cada empresa de inserción aplicaba en las tres fases del itinerario de

Gráfico 1. Distribución de las empresas de inserción de la muestra, según comunidad autónoma de procedencia (\%)

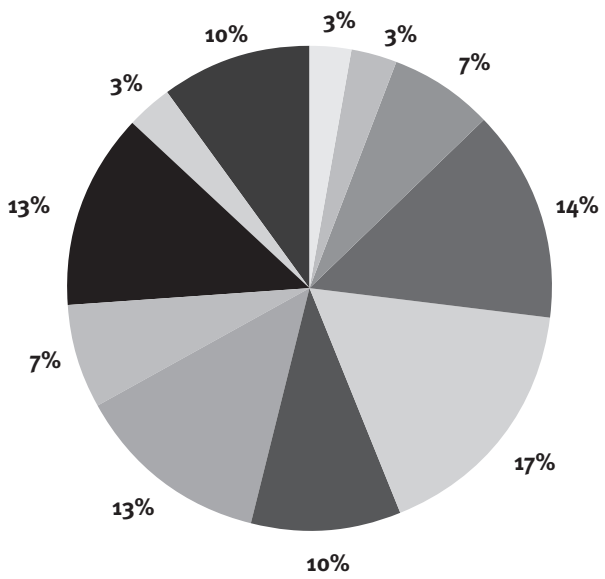
La Rioja
Cataluña
Principado de Asturias
Illes Balears
Castilla-La Mancha
Canarias
Com. Valenciana
Región de Murcia
Castilla-León
País Vasco
Com. de Madrid

Fuente: Zacarés et al. (2011). inserción. FAEDEI y AERESS contactaron así con sus entidades asociadas y gestionaron la recogida de los instrumentos demandados, para su posterior envío al equipo de investigación de la Universitat de València. Sesenta de las empresas de inserción contactadas enviaron algún tipo de información, si bien únicamente se recibieron instrumentos de valoración de la mitad de ellas. En el Gráfico 1, se presentan las treinta empresas de las que se recogieron instrumentos, distribuidas por comunidades autónomas.

Todos los instrumentos recogidos se solicitaron a las empresas, pidiéndoles al mismo tiempo que los enmarcasen en una de las fases generales del itinerario de inserción. Los instrumentos de acogida se refieren a todo tipo de instrumentos utilizados en el momento de ingreso del trabajador de inserción en la empresa. Los instrumentos de seguimiento son aquellos utilizados con el fin de evaluar el proceso que sigue cada trabajador de inserción durante el periodo de permanencia en la empresa. Y, por último, los instrumentos de transición se refieren a los utilizados para evaluar el proceso seguido, así como la adecuación final del trabajador a la empresa ordinaria (Zacarés et al., 2011). En la Tabla 1 se presentan los instrumentos recogidos, según las distintas fases, en función de la comunidad autónoma de procedencia.

Se utilizó el Instrumento para el Análisis de los Indicadores de Empleabilidad (IPAIE), de Llinares, Cordoba y Zacarés (2011). Esta herramienta -creada a partir de la estructuración realizada, entre otros, por McQuaid y Lindsay (2005), y Navarro et al. (2008)-, presenta y clasifica todos los indicadores de la empleabilidad que, hasta el momento, se han presentado en la literatura sobre el tema. Tal y como puede observarse en el Cuadro 1, los indicadores de empleabilidad se estructuran en torno a tres ejes: factores individuales, características personales y factores externos.

Los factores individuales recogen todos aquellos indicadores que se refieren a variables actitudinales o aptitudinales que están relacionadas con las competencias laborales. En la categoría de circunstancias personales, se recogen aquellos aspectos sociofamiliares que inciden en los factores individuales y que determinan la capacidad de integración de la persona en el mundo laboral. Los factores externos incluyen indicadores del mercado de trabajo de cada contexto concreto, así como dimensiones del macrosistema que inciden en la consecución y mantenimiento del empleo.

En el estudio llevado a cabo en Llinares, Córdoba y Zacarés (2011), se analizaron todos los instrumentos que han desarrollado las empresas de inserción españolas anteriormente descritos. En este estudio se ha analizado qué se mide y desde qué perspectiva en los instrumentos elaborados por empresas localizadas en el País Vasco; se pretende, pues, profundizar en el ámbito de la inserción laboral en un territorio que ha mostrado una especial sensibilidad social y política al respecto. 
Tabla 1. Instrumentos analizados, por comunidad autónoma de origen y fase a la que se asignaron

\begin{tabular}{|c|c|c|c|c|c|c|}
\hline & \multicolumn{5}{|c|}{ Instrumentos } & \multirow{3}{*}{ Empresas de inserción } \\
\hline & \multicolumn{3}{|c|}{ Por fase } & \multirow{2}{*}{ Protocolos } & \multirow{2}{*}{ Total } & \\
\hline & Acogida & Seguimiento & Final & & & \\
\hline Canarias & 8 & 8 & 2 & 2 & 20 & 4 \\
\hline Castilla-La Mancha & 3 & 15 & 2 & 7 & 27 & 2 \\
\hline Castilla-León & 15 & 7 & 4 & 5 & 31 & 5 \\
\hline Cataluña & 18 & 18 & 5 & 6 & 47 & 4 \\
\hline Com. de Madrid & 5 & 5 & 3 & 1 & 14 & 3 \\
\hline Com. Valenciana & 16 & 25 & 2 & 2 & 45 & 4 \\
\hline Illes Balears & 6 & 8 & 0 & 0 & 14 & 2 \\
\hline La Rioja & 3 & 6 & 1 & 2 & 12 & 1 \\
\hline País Vasco & 4 & 6 & 1 & 0 & 11 & 3 \\
\hline Principado de Asturias & 4 & 9 & 1 & 4 & 18 & 1 \\
\hline Región de Murcia & 4 & 5 & 1 & 0 & 10 & 1 \\
\hline Total & 86 & 112 & 22 & 29 & 249 & 30 \\
\hline
\end{tabular}

Fuente: Zacarés et al. (2011).

Cuadro 1. Indicadores de la empleabilidad: ejes estructuradores e indicadores primarios

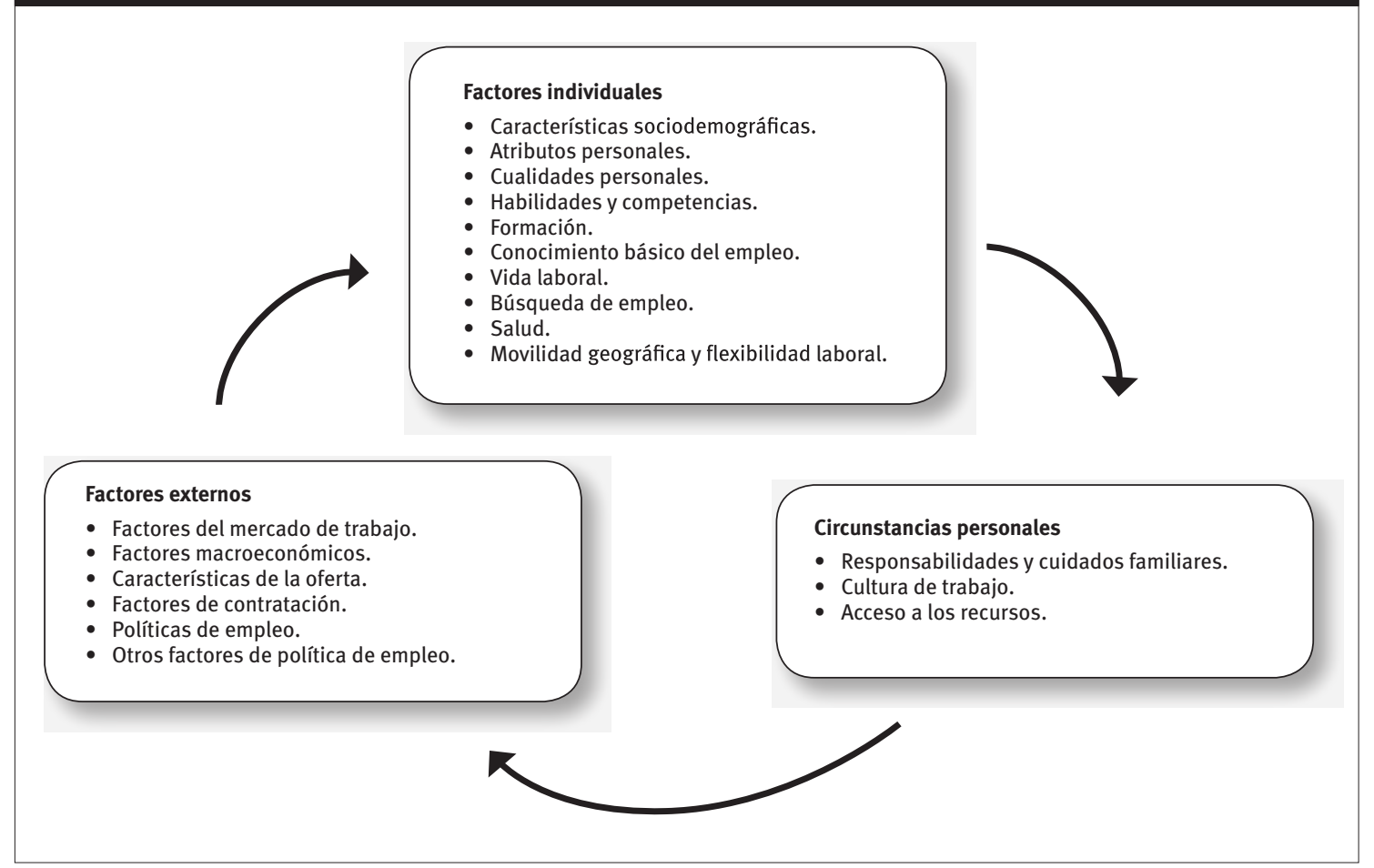

Fuente: Elaboración propia.

\section{3. ¿Qué medimos cuando evaluamos la empleabilidad?}

La primera pregunta que nos planteamos en el análisis de la medida de la empleabilidad hace referencia a qué indicadores tienen en cuenta las personas que trabajan en las empresas de inserción cuando la evalúan. El Cuadro 2 muestra los porcentajes de indicadores en función de los ejes estructuradores para todos los instrumentos que se remitieron desde las empresas de inserción localizadas en el País Vasco, sin tener en cuenta las fases de inserción. Cabe señalar que, al igual que sucede en el resto de España, los instrumentos con los que se lleva a cabo el análisis de la empleabilidad miden, sobre todo, factores individuales y, en menor medida, circunstancias personales. 


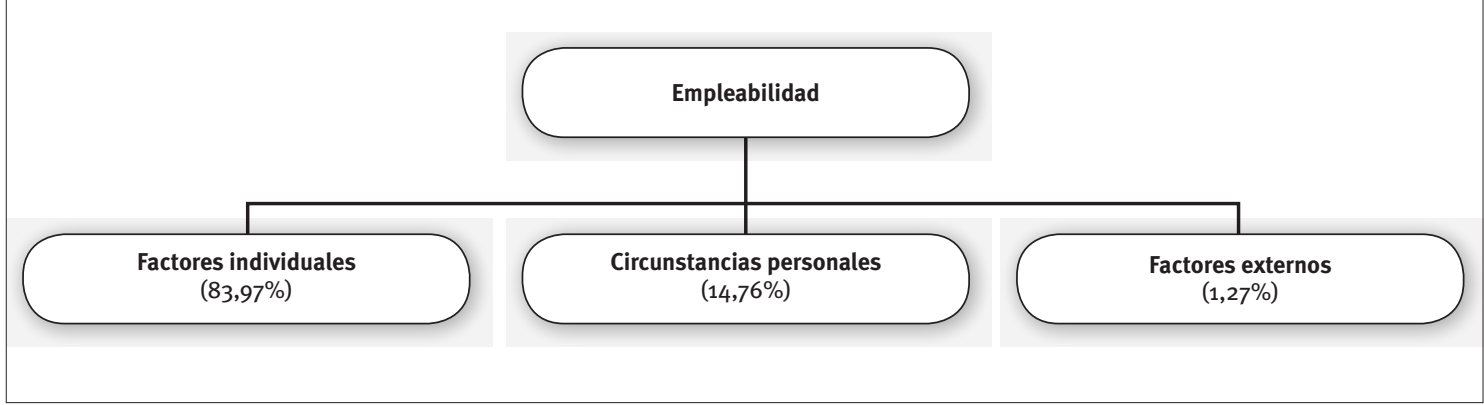

Fuente: Elaboración propia.

El mayor porcentaje referido a los factores individuales muestra que lo que medimos en nuestra población en riesgo de exclusión son, sobre todo, aspectos relativos al sujeto. De entre ellos, tal y como muestran los Gráficos 2 y 3, se analizan las habilidades y competencias, y los atributos personales. La categoría de habilidades y competencias es la más amplia dentro de los factores individuales. Los aspectos más analizados por las distintas empresas en esta fase de acogida son, al igual que el resto de empresas de inserción de España, las habilidades y competencias personales generales, así como las habilidades sociales. Los indicadores que más se tienen en cuenta en las habilidades y competencias personales generales son la iniciativa, la independencia y la autoorganización (en la gestión del tiempo y las tareas). Con respecto a las habilidades sociales, se miden, sobre todo, el trabajo en equipo, la aceptación de críticas, las habilidades sociales básicas de comunicación interpersonal y la gestión del conflicto.

Con respecto a la categoría de atributos personales, cabe señalar que no es tan amplia como la de habilidades y competencias, pero recoge más o menos el $19 \%$ de los elementos que se preguntan para evaluar la empleabilidad. En ella, las cualidades personales son las que aparecen en mayor medida en los instrumentos de evaluación y, entre éstas, cabe destacar la autoestima, la tolerancia y el respeto, la tolerancia al estrés y la frustración, y la responsabilidad.

La categoría de características sociodemográficas está presente en casi todos los cuestionarios y tiene en cuenta indicadores que preguntan, sobre todo, por la edad, el sexo, la nacionalidad, el estado civil y la situación legal administrativa. Aunque en menor grado, también parece relevante para la medida de la empleabilidad las cuestiones relativas a: a) la salud, sobre todo en lo que concierne a salud física y dependencias; b) la formación académica reglada y no reglada; c) el conocimiento básico del empleo, sobre todo en lo que hace referencia a las habilidades profesionales específicas; y d) las técnicas de búsqueda de empleo. Menos relevante, aunque se ha tenido en cuenta, parece resultar la vida laboral. No obstante, sí se consideran la experiencia laboral en el mercado normalizado, la movilidad geográfica, la flexibilidad laboral y la disponibilidad de la persona.

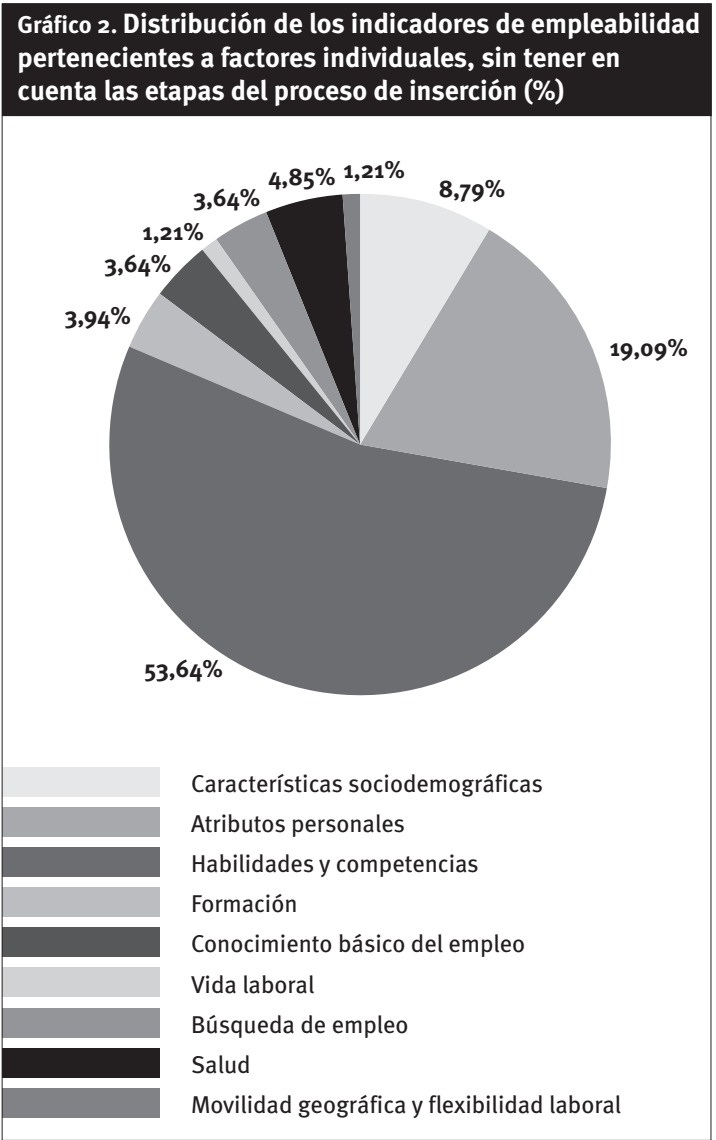

Fuente: Elaboración propia.

Tal y como se ha comentado anteriormente, en las empresas de inserción la empleabilidad se mide en tres momentos que tienen como telón de fondo las fases generales del itinerario de inserción (acogida, seguimiento, y transición o tránsito al mercado laboral ordinario). Resulta interesante observar que, en la fase de acogida, cuando se lleva a cabo la valoración inicial que incluye el diagnóstico de empleabilidad, hay más dispersión de indicadores, lo que nos muestra que se evalúan aspectos muy diversos de la persona. Sin embargo, cabe resaltar la gran cantidad de habilidades y competencias que resultan relevantes para conocerla. 
Fuente: Elaboración propia.

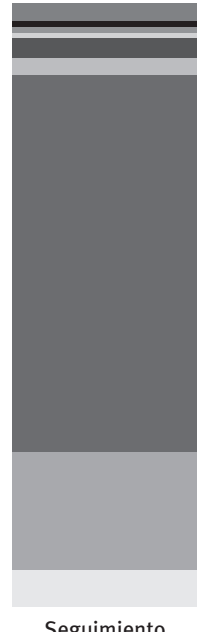

Seguimiento

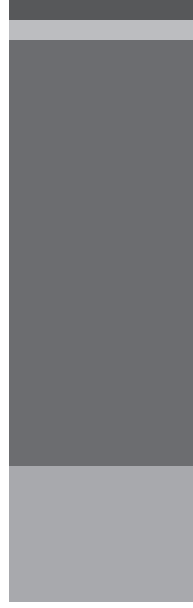

Evaluación

Características sociodemográficas

Atributos personales

Habilidades y competencias

Formación

Conocimiento básico del empleo

Vida laboral

Búsqueda de empleo

Salud

Movilidad geográfica y flexibilidad laboral

El Gráfico 3 también nos muestra un hecho muy curioso: en la fase de seguimiento, se acompaña al trabajador en el desempeño de un puesto de trabajo; en las empresas de inserción, esto supone su capacitación laboral, pero también personal y social, y, por tanto, el seguimiento de la empleabilidad. Como puede observarse, los instrumentos creados a tal efecto por las entidades se centran, sobre todo, en habilidades y competencias. Aunque en esta fase también tienen presencia otros indicadores generales, casi el $83 \%$ de ellos se aglutinan en habilidades, competencias y atributos personales. Lo mismo sucede en la fase de transición. En ésta, los instrumentos valoran la empleabilidad del trabajador para su incorporación en una empresa en el mercado laboral ordinario, la cual parece depender de las habilidades y competencias (71\%) y, también en parte, de los atributos personales (22,6\%).

Las circunstancias personales como indicadores que completan la medida de la empleabilidad incluyen todos aquellos aspectos que repercuten en la persona, condicionando su posición en el mundo laboral, pero que no son propios de ella misma. Tal y como se puede observar en la Tabla 2, el acceso a los recursos es el elemento más relevante de las circunstancias personales en cuanto a la medida de la empleabilidad se refiere.

De entre los aspectos considerados relevantes en la valoración de la empleabilidad, cabe resaltar la importancia del acceso a redes de apoyo, y la variedad y número de éstas. Además, se tiene en cuenta la situación económica y, en concreto, se pregunta por el acceso a fuentes formales/informales de apoyo financiero (prestaciones sociales). El transporte y la vivienda también son considerados importantes para el acceso al empleo. En cuanto al elemento 'hijos', se tiene en cuenta en gran parte de los cuestionarios como una categoría de las circunstancias personales que condiciona la empleabilidad. No sucede lo mismo, por ejemplo, con las cargas familiares de padres ancianos, ni se presta especial atención a la estructura familiar.

Con respecto a los factores externos, y tal y como muestra la Tabla 3, cabe señalar que las empresas de inserción no los consideran en la valoración inicial de la empleabilidad. Los aspectos que se han tenido en cuenta en uno de los cuestionarios hacen referencia a la remuneración y a las condiciones de trabajo. 
Tabla 2. Frecuencias de los indicadores que pertenecen a la categoría de circunstancias personales

\begin{tabular}{l|l|c|c|c|c}
\cline { 2 - 5 } & \multicolumn{1}{|c|}{ Aspectos más relevantes } & Total & \multicolumn{2}{c}{ Fases } \\
\cline { 4 - 6 } & & & Acogida & Seguimiento & Evaluación \\
\hline $\begin{array}{l}\text { Responsabilidades, cuidados } \\
\text { familiares }\end{array}$ & $\bullet$ Hijos. & 10 & 7 & 3 & 0 \\
\hline Cultura del trabajo & & 0 & 0 & 0 & 0 \\
\hline Acceso a recursos & & 24 & 24 & 0 & 0 \\
\hline Transporte y vivienda & $\begin{array}{l}\text { - Régimen vivienda. } \\
\text { - Acceso a transporte privado o } \\
\text { fácilmente disponible. }\end{array}$ & 7 & 7 & 0 & 0 \\
\hline Situación económica & $\begin{array}{l}\text { - Acceso a fuentes formales/informales } \\
\text { de apoyo financiero. }\end{array}$ & 8 & 8 & & 0 \\
\hline Apoyo social & $\begin{array}{l}\text { - Acceso a redes de apoyo. } \\
\text { Número, variedad y estado de las redes } \\
\text { de apoyo. }\end{array}$ & 9 & 9 & & 0 \\
\hline
\end{tabular}

Fuente: Elaboración propia.

Tabla 3. Frecuencias de los indicadores que pertenecen a la categoría de factores externos

\begin{tabular}{|c|c|c|c|c|c|}
\hline & \multirow{2}{*}{ Aspectos más relevantes } & \multirow{2}{*}{ Total } & \multicolumn{3}{|c|}{ Fases } \\
\hline & & & Acogida & Seguimiento & Evaluación \\
\hline Factores del mercado de trabajo & & 0 & 0 & 0 & 0 \\
\hline Factores macroeconómicos & & 0 & 0 & 0 & 0 \\
\hline Características de la oferta & $\begin{array}{l}\text { - Remuneración. } \\
\text { - Condiciones del empleo. } \\
\text { - Horas de trabajo y turnos. }\end{array}$ & 4 & 0 & 4 & 0 \\
\hline Factores de contratación & & 0 & 0 & 0 & 0 \\
\hline Políticas de empleo & & 1 & 1 & 0 & 0 \\
\hline Otros factores de política de empleo & & 0 & 0 & 0 & 0 \\
\hline
\end{tabular}

Fuente: Elaboración propia.

Hasta el momento, se ha llevado a cabo un análisis en función de las categorías en las que se clasifican los indicadores de empleabilidad; con ello conseguimos saber qué cualidades debe poseer una persona empleable para los profesionales que trabajan en las empresas de inserción. Ahora bien, para conocer el núcleo central de la representación de la empleabilidad, necesitamos examinar los elementos de la empleabilidad en los que la mayor parte coinciden. Para ello, se han estudiado los indicadores que tienen una incidencia superior al $65 \%$. Si se tienen en cuenta los indicadores más utilizados por la mayor parte de las empresas, cabe señalar que, como se observa en el Cuadro 3, los cuestionarios coinciden en valorar aspectos individuales del sujeto. La mayor parte de ellos consideran la autoestima, la iniciativa, el actuar de forma autónoma, el trabajo en equipo y las habilidades profesionales específicas como elementos clave de la empleabilidad. Más del $80 \%$ de ellos también resaltan la importancia de la tolerancia al estrés y la frustración, la tolerancia y el respecto, la autoorganización, la aceptación de críticas y el aprender a aprender. También resultan relevantes en la medida de la empleabilidad la edad, el sexo, el cuidado personal, la autoeficacia, la capacidad de lectoescritura y de comunicación oral y escrita, la autodisciplina, la perseverancia, la proactividad, las habilidades sociales básicas de comunicación interpersonal y la asertividad, la gestión positiva del conflicto, la toma de decisiones, y las cualificaciones académicas y profesionales formales.

Así pues, parece que los profesionales que trabajan en las empresas de inserción tienen una representación de la empleabilidad como competencia individual cuyos indicadores principales y comunes son algunas habilidades y competencias generales (dentro de las que se encuentran, sorprendentemente, la iniciativa y la autonomía, habilidades sociales (se observa la actual importancia del trabajo en equipo en nuestro modo de comprender la realidad laboral) y algo de conocimiento básico del empleo, en concreto, las habilidades profesionales específicas. Este último contenido del núcleo central de la representación de la empleabilidad dista mucho de los anteriores indicadores de la empleabilidad. Mientras iniciativa y autonomía son básicas para la adaptación a puestos de trabajo cambiantes y difusos, el conocimiento concreto de un puesto de trabajo resulta crucial en la etapa del Estado del bienestar en donde se pretendía el ajuste entre personas y puestos de trabajo. De esta forma, se observa cómo las lógicas legitimadoras de la nueva forma de comprender el trabajo y la situación social de la persona nos inducen a tener discursos cuyos elementos pueden llegar a ser contradictorios, porque su universo simbólico no excluye los contenidos de las anteriores imágenes de los requerimientos laborales. 
Características sociodemográficas

- Edad.

- Sexo.

Atributos personales

- Cuidado personal.

- Cualidades personales: responsabilidad, tolerancia al estrés y la frustración, seguridad en uno mismo, autoeficacia, autoestima, y tolerancia y respeto.

Habilidades y competencias

- Habilidades básicas: capacidad básica de lectoescritura, cálculo, capacidad para la comunicación oral y escrita.

- Hábitos laborales básicos: puntualidad.

- Habilidades y competencias personales generales: autodisciplina, iniciativa, actuar de forma autónoma, perseverancia, gestión del tiempo y de las tareas.

- Adaptabilidad: proactividad.

- Habilidades sociales: habilidades sociales básicas de comunicación interpersonal, aceptación de críticas, gestión positiva del

conflicto, trabajo en equipo.

- Potencial de aprendizaje: aprender a aprender.

- Análisis de situaciones y toma de decisiones: toma de decisiones.

\section{Formación}

- Calificaciones académicas y profesionales formales.

Conocimiento básico del empleo

- Habilidades profesionales específicas.

Fuente: Elaboración propia.

\section{La representación de la empleabilidad desde las empresas de inserción del País Vasco y su evaluación de los colectivos vulnerables}

Las representaciones sobre la empleabilidad proporcionan nociones y teorías estables y eficaces sobre las características del desempleado, y el motivo de su situación y de las relaciones que establece con su entorno. Este proceso tiene repercusiones en la estigmatización y categorización de las personas vulnerables que, a menudo, son vistas, y se ven a sí mismas, como responsables de la situación que están viviendo.

En este artículo no se pretende ni responsabilizar ni dejar de responsabilizar a la persona de sus circunstancias; su objetivo es el análisis de la concepción de empleabilidad que tienen los agentes sociales implicados en la inserción laboral de colectivos vulnerables $y$, en concreto, que trabajan en empresas de inserción del País Vasco. El porqué del estudio recae en la influencia que tiene la evaluación en las variables de la persona que influyen en el afrontamiento de situaciones estresantes y los mecanismos que activan para salir de ellas. Como la representación social actúa como ‘atajo' mental, no permite el análisis racional e individual de las características de cada persona, lo que implica que no se atienda a su singularidad, sino a sus características como miembro del grupo social al que pertenece: empleable/no empleable. El artículo también hace una llamada al análisis de las prácticas en función de las teorías implícitas sobre las que se basan, y de las que, a menudo, no somos conscientes.
Para alcanzar el citado objetivo, se ha realizado un estudio sobre los indicadores que las empresas de inserción incluyen en las evaluaciones de la empleabilidad en los tres momentos del proceso. Parece que la empleabilidad se mide a partir del análisis de, sobre todo, los factores individuales; junto a ello, las circunstancias personales completan la medida de la empleabilidad. De entre los factores individuales, la empleabilidad parece concretarse en determinadas habilidades y competencias, atributos personales, características sociodemográficas, de búsqueda de empleo y de salud. Aunque algunas entidades, en sus instrumentos, también reflejan que la empleabilidad debe evaluarse a partir de indicadores que reflejen la formación, la vida laboral, la movilidad geográfica y la flexibilidad laboral, así como el conocimiento básico del empleo. De entre las circunstancias personales, parece que las que se tienen en cuenta son el acceso a los recursos, y las responsabilidades y cuidados familiares.

Este panorama requiere retomar la meta de toda empresa de inserción: la integración y formación sociolaboral de personas en situación de exclusión social como tránsito al empleo ordinario. En ese intento de inclusión en el mercado normalizado de trabajo, las empresas de inserción tienen que evaluar la empleabilidad. La empleabilidad proporciona la base discursiva que legitima la desigualdad social, en general, y de las clases vulnerables y en riesgo de exclusión laboral y social, en particular. Desde la perspectiva posibilista de la empleabilidad, la inclusión/ exclusión de una persona depende del desarrollo de ciertas características personales. Desde esa base, se plantea la nueva psicología del trabajo, que se 
centra en la persona y no en sus circunstancias ni en el mercado laboral; que se ocupa de las competencias y no de los puestos de trabajo; que pasa de considerar la empresa como la principal creadora de las carreras laborales y de la estabilidad en el empleo, a percibirla como potenciadora de los proyectos de futuro de los trabajadores y camino de paso de trabajadores en búsqueda de su desarrollo. Es por eso por lo que, tal y como hemos podido observar en los resultados obtenidos, la representación de la empleabilidad tiene en su núcleo aspectos como la iniciativa y la autonomía, que podrían ser considerados 'competencias de alto nivel de complejidad', propios de profesionales de elevada cualificación, y no tanto de colectivos excluidos del mundo laboral.

Junto a ello, y partiendo del modelo ecológico de Bronfenbrenner (Bronfenbrenner y Morris, 1998), que contempla a la persona en su contexto, consideramos que las circunstancias personales y los factores exteriores son parte fundamental para el análisis de la empleabilidad. La representación de la empleabilidad tiene en cuenta algunos indicadores referidos a las circunstancias personales y presupone el grado de empleabilidad considerando las responsabilidades familiares y el acceso a los recursos. De esta forma, la percepción individualista de la empleabilidad queda mediatizada por su historia individual y social.

No sucede lo mismo con los factores externos. Parece, pues, que la representación de la empleabilidad no contempla el contexto social, político y laboral que la enmarca. Es percibida ajena a aspectos económicos, como los nuevos modos de contrata- ción; políticos, como las políticas de ocupabilidad; socioculturales, como la relación entre las redes sociales y el empleo; y tecnológicos, como Internet. De este modo, parece que se conceptualiza la empleabilidad, sobre todo, desde variables individuales que tienen en cuenta algunas relacionales, en concreto, aquellas que se vinculan directamente al microsistema del sujeto.

Nos situamos, pues, ante una percepción de la realidad acorde con un prototipo de trabajador (el del alto ejecutivo), cuya presencia en la sociedad actual es mínima, y que es contemplada desde una perspectiva cognitiva que desconsidera el contexto. $Y$ es en ese marco en el que las empresas de inserción se plantean como un instrumento para la integración de aquellos colectivos que parecen no tener un lugar en el mundo laboral. Sin embargo, a ese colectivo vulnerable, cuyos recursos están muy alejados de los del alto ejecutivo, se le exigen determinadas habilidades y competencias que no le son propias y resultan difícilmente accesibles para él.

Todas las cuestiones que se han planteado pretenden aportar un primer acercamiento analítico a una realidad relativamente nueva, que requiere mayor sistematización, tanto por parte de los investigadores como de los agentes sociales, para quienes resulta ser un instrumento de trabajo. La medida de la empleabilidad requiere de las empresas de inserción continúen la reflexión conjunta que permita su uso en función de los fines sociales para los que fueron creadas, y amplíen su concepción a otras instancias que no sean únicamente el individuo. 
ALALUF, M.; y MARTÍNEZ, E. (1999): “Bélgica: el empleo desestabilizado por el desempleo. Empleo precario y desempleo activo", en PRIETO, C. (ed.), La crisis del empleo en Europa, Alzira, Germania.

ÁLVAREZ-URÍA, F. (2002): "Estado social versus neoliberalismo", Acciones e Investigaciones Sociales, $\mathrm{n}$ ำ16, págs. 13-23 [<http://www. unizar.es/centros/eues/html/archivos/ temporales/16_AIS/AIS_16\%2805\%29.pdfs].

AÑEZ, C. (2004): “El Estado del bienestar social y el neoliberalismo ante los derechos laborales", Revista de Ciencias Sociales, vol. X, n- 1, págs. 70-82 [<http://redalyc.uaemex.mx/src/inicio/ ArtPdfRed.jsp?iCve=28010106〉].

BARUCH, Y.; y PEIPERL, M. (2000): “Career management practices: An empirical survey and implications", Human Resource Management, vol. 39, no 4, págs. 347-366 [shttp://tinyurl. com/6nvylkg)]

BRONFENBRENNER, U.; y MORRIS, P. A. (1998): "The ecology of developmental processes", en DAMON, W.; y LERNER, R. M. (eds.), Handbook of Child Psychology. Vol. 1. Theoretical Models of Human Developments, Nueva York, John Wiley and Sons.

BRUNET, I.; y PASTOR, I. (2003): “Tercera vía, comisión europea y estrategias de empleabilidad", Anduli, no 3, págs. 11-24 [<http:// dialnet.unirioja.es/servlet/dcfichero_ articulo? codigo $=1973117>$ ).

CAMPOS RÍOS, G. (2003): “Implicaciones económicas del concepto de empleabilidad", Aportes, vol. 8, no 23, págs. 101-111 [rhttp://redalyc.uaemex. $\mathrm{mx} /$ redalyc/pdf/376/37602308.pdf〉].
CAPPELLI, P. (1995): “Rethinking employment”, British Journal of Industrial Relations, vol. 33, nํㅜ 4 .

CASAL, J. (2003): “La transición de la escuela al trabajo", en FERNÁNDEZ PALOMARES, F, (coord.), Sociología de la educación, Madrid, Pearson Educación, págs. 179-200.

CASTELLS, M. (2001): La era de la información. Vol. III. Fin del milenio, México, Siglo XXI.

COMISIÓN EUROPEA (2003): Comunicación de la Comisión al Consejo, al Parlamento Europeo, al Comité Económico y Social y al comité de las Regiones. El futuro de la Estrategia Europea de Empleo (EEE): "Una estrategia para el pleno empleo y mejores puestos de trabajo para todos", Bruselas, Comisión Europea [shttp://eur-lex. europa.eu/LexUriServ/LexUriServ.do?uri=COM: 2003:0006:FIN:ES:PDF>].

- (1999): The European Employment Strategy: Investing in People; Investing in More and Better Jobs, Luxemburgo, Oficina de Publicaciones de la Unión Europea.

CLARKE, M.; y PATRICKSON, M. (2008): "The new covenant of employability”, Employee Relations, vol. 30 no 2, págs. 121-141.

DE CUYPER, N. et al. (2011): "The role of job resources in the relation between perceived employability and turnover intention: A prospective two-sample study", Journal of Vocational Behavior, vol. 78, no 2, págs. 253-263.

DE LA FUENTE, R.; y GONZÁLEZ-CASTRO, J. L. (2009): "Empleo, formación e inserción de colectivos en riesgo de exclusión. Un reto social y económico para la empresa receptora", Bordón. Revista de Pedagogía, vol. 61, no-3, págs. $33-46$ [<http://dialnet.unirioja.es/servlet/dcfichero_ articulo? codigo $=3054918$ > . 
FINN, D. (2000): "From full employment to full employability: A new deal for Britain's unemployed?", International Journal of Manpower, nํㅜ 21, págs. 384-399.

FORMICHELLA, M. M.; y LONDON, S. (2005): Reflexiones acerca de la noción de empleabilidad, Buenos Aires, Asociación Argentina de Economía Política [<http://www.aaep.org.ar/anales/ works/works2005/formichella_london.pdf〉].

FORRIER, A.; SELS, L.; y STYNEN, D. (2009): “Career mobility at the intersection between agent and structure: A conceptual model", Journal of Occupational and Organizational Psychology, vol. 82, nํㅜ 4, págs. 739-759.

FUGATE, M.; KINICKI, A. J.; y ASHFORTH, B. E. (2004): "Employability: A psycho-social construct, its dimensions, and applications", Journal of Vocational Behavior, vol. $65, \mathrm{n}^{\circ}$ 1, págs. $14-38$ [<http://dx.doi. org/10.1348/096317909X470933)]

GONZÁLEZ BARRIGA, J. R. (2003): “Introducción y marco jurídico de las empresas de inserción laboral”, Acciones e Investigaciones Sociales, $\mathrm{n}-18$, págs. 89-101 [khttp://www.unizar.es/centros/ eues/html/archivos/temporales/18_AIS/AIS$18 \% 2805 \% 29$.pdf $>$ ].

HARRISON, B. (1998): "Labor market restructuring and workforce development”, en GILOTH, R. P. (ed.), Jobs and Economic Development, Londres, Sage.

KIPPENBERGER, T. (2000): "The scouring effect of change", The Antidote, $\mathrm{n}-5$, págs. $18-20$.

LLINARES, L.; CÓRDOBA, A.; y ZACARÉS, J. J. (2011): "La medida de la empleabilidad desde las empresas de inserción social”, en CÓRDOBA, A.; y MARTÍNEZ MORALES, I. (coords.), Trabajo, empleabilidad y vulnerabilidad social: condicionantes y potencialidades de la integración a través de las empresas de inserción social, Valencia, Servicio de Publicaciones de la Universitat de València.

LUTTWAK E. (2000): Turbocapitalismo. Quiénes ganan y quiénes pierden en la globalización, Barcelona, Crítica.

MACÍA, M. C. (2003): "Neoliberalismo y relaciones de trabajo", en KURCZY, P.; y PUIG HERNÁNDEZ, C. A. (coords.), Estudios jurídicos en homenaje al Doctor Néstor de Buen Lozano, México, Universidad Nacional Autónoma de México.

MARHUENDA, F. (2009): "Formación y empleabilidad en las empresas de inserción social”. Comunicación presentada en el V Congreso Internacional de Formación para el Trabajo. 24/26 de junio de 2009, Granada.

MARHUENDA, F.; BERNAD, J. C.; y NAVAS, A (2010): “La práctica en empresas como estrategia de enseñanza e inserción laboral: las empresas de inserción social”, Revista de Educación, no 351, págs. 139-161 [<http://www.revistaeducacion. educacion.es/re351/re351_06.pdf〉]. employability”, Urban Studies, vol. 42, no 2 , págs. 197-219.
MINGLIONE, E. (1994): Las sociedades fragmentadas. Una sociología de la vida económica más allá del paradigma del mercado, Madrid, Ministerio de Trabajo y Seguridad Social.

NAVARRO, J. A. et al. (2008): Cuestionario Sociométrico de la Empleabilidad, Valencia, Pactem Nord.

NOGUERA, J. A. (2002): “El concepto de trabajo y la teoría social crítica”, Papers, no 68, págs. 141-168 [<http://www.raco.cat/index.php/papers/ article/viewFile/25717/25551)].

OSTERMAN, P. (1994): "How common is workplace transformation and who adopts it?", Industrial and Labor Relation Review, vol. 47, no- 2.

PÉREZ, C. (2003): “El discurso de la empleabilidad y las funciones del sistema educativo. Repercusiones para la sociología de la educación". Comunicación presentada en la X Conferència de Sociologia de l'Educació, Valencia, 18-20 de septiembre de 2003.

PRIETO, C. (1999a): "Crisis del empleo, ¿crisis del orden social?”, en MIGUÉLEZ, F.; y PRIETO, C. (eds.): Las relaciones de empleo en España, Madrid, Siglo XXI.

- (ed.) (1999b): La crisis del empleo en Europa, Alzira, Germania.

ROTHERY, B.; y ROBERSTSON, I. (1995): The Truth about Outsourcing, Nueva York, Gower Publishing.

SANTOS ORTEGA, A. (2003): "La invasión de la ideología empresarial en los márgenes del mercado de trabajo: inseguridad laboral, paro e inserción", Cuadernos de Relaciones Laborales, $\mathrm{n}-21$, págs. 107-128.

TEZANOS, J. F. (2001): El trabajo perdido. ¿Hacia una civilización postlaboral?, Madrid, Biblioteca Nueva.

THUROW, L. (2000): Construir riqueza, Buenos Aires, Javier Vergara Editor.

VAN DER HEIJDE, C. M.; y VAN DER HEIJDEN, B. I. J. M. (2005): "The development and psychometric evaluation of a multidimensional measurement instrument of employability-and the impact of aging", International Congress Series, vol. 1.280, págs. 142-147.

WEINBERG, P. D. (2004): “Formación profesional, empleo y empleabilidad". Ponencia presentada al Foro Mundial de Educación, Porto Alegre, julio [shttp://ilo-mirror.library.cornell.edu/public/ spanish/region/ampro/cinterfor/publ/sala/ weinberg/index.htm〉].

ZACARÉS, J. J. et al. (2011): ·Análisis de los instrumentos de valoración empleados por las empresas de inserción social. Una visión desde las fases del proceso", en CÓRDOBA, A.; y MARTÍNEZ MORALES, I. (coords.), Trabajo, empleabilidad y vulnerabilidad social: condicionantes y potencialidades de la integración a través de las empresas de inserción social, Valencia, Servicio de Publicaciones de la Universitat de València. 